\title{
LA CIUDAD COMPARTIDA
}

IDEAS SOBRE LA NECESIDAD DEL DESARROLLO DE POLÍTICAS PÚBLICAS PARA LOGRAR UN DESARROLLO SOSTENIBLE DE LAS CIUDADES EN LOS PAÍSES EN VÍAS EN DESARROLLO (O EN LAS MÁS VULNERABLES), SOBRE LA BASE DEL CONCEPTO DE LOS BIENES COMUNES (URBANOS) Y DE LA IDEA DE ECONOMÍA COLABORATIVA QUE SUBYACE EN LA NATURALEZA DE SU GESTIÓN)

\author{
Teresa Parejo Navajas \\ Profesora Contratada Doctora \\ Universidad Carlos III de Madrid
}

\section{1.- Planteamiento:}

Los bienes comunes son aquellos que pertenecen a la colectividad y, por tanto, son de todos y de nadie al mismo tiempo. Su protección constitucional -referida, en principio, solo a bienes materiales; así, por ejemplo, en el artículo 132 de la Constitución española (los llamados bienes públicos por naturaleza, especialmente las aguas continentales y la costa y el mar territorial)-, pretende otorgarles la mayor garantía jurídica para que no estén al albur de los gobiernos de turno. Ocurre que esta garantía está lastrada en muchos casos (por ejemplo en España) por la concepción de que el "dominio público" no es tanto una propiedad fiduciaria del Estado, como una propiedad a secas y, por ello, cabe la desafectación de bienes concretos, que -convertidos así en bienes patrimoniales- pueden ser enajenados (privatizados). Lo público (dominado por el

WPS RI-SHUR, n4, 2016, vol.1, ISSN: 2387-1768 


\section{WPSReview International on Sustainable Housing and Urban Renewal}

(RI-SHUR)

Estado) y lo privado (dominado por las grandes corporaciones ), en aparente contradicción, son en realidad lo mismo, pues tanto uno como otro tienen su interés en el mercado. ${ }^{1}$

Por eso, y siguiendo al profesor Mattei ${ }^{2}$, las privatizaciones que de estos bienes se ha venido realizando a lo largo de los últimos tiempos, como deriva lógica del "triunfo en Occidente del constitucionalismo liberal", ${ }^{3}$ suponen una injerencia contra legem en aquéllo que pertenece a cada miembro de la comunidad, siempre que se interprete correctamente la normativa constitucional como propiedad fiduciaria (a cuyo efecto es preciso combatir la interpretación vigente hasta lograr un tratamiento de los bienes correspondientes como verdaderamente pertenecientes a todos, según la idea original romana y en la línea de la doctrina estadounidense de la public trust).

El concepto de los "commons" o bienes comunes (ahora ya en un sentido más amplio), puede proporcionar las herramientas políticas y legales recuperar la defensa de la justicia social, dando poder a la población para adoptar acciones directas para su protección. ${ }^{4}$ Por ello, los bienes comunes, como marco institucional, se constituyen como paradigma legal y político alternativo al actualmente vigente a los efectos de proveer una distribución de los recursos más equitativa y, por tanto, una mayor justicia social. ${ }^{5}$

En esta línea se hace preciso, además, trabajar a fin de lograr la extensión de los bienes comunes a todos cuantos, en la sociedad de hoy, deban ser considerados pertenecientes a la colectividad, en particular -dada la importancia actual del medio urbano- los que se inscriben en el mundo de las ciudades.

A este escenario se añaden nuevos retos -numerosos y complejos, de los que se extraen cuatro, especialmente relevantes en lo que aquí interesa- que urgen al replanteamiento del actual sistema de organización socio-económica de las actividades del hombre:

\footnotetext{
${ }_{1}^{1}$ Matteri, Ugo. "The State, the market, and Some Preliminary Questions about the Commons (french and English vesrions)." University of California, Hasting College of Law. FRom the SElected Works of Ugo Mattei, March 18, 2011.

2 Mattei, Ugo, Bienes Comunes. Un Manifiesto, Editorial Trotta, 2011.

3 Id., p. 10.

${ }^{4}$ Matteri, Ugo. "The State, the market, and Some Preliminary Questions about the Commons (french and English vesrions)." University of California, Hasting College of Law. FRom the SElected Works of Ugo Mattei, March 18, 2011.

${ }^{5}$ Id.
}

WPS RI-SHUR, n4, 2016, vol.1, ISSN: 2387-1768

NÚMERO MONOGRÁFICO SOBRE GOBERNANZA URBANA/SPECIAL ISSUE ON GOVERNANCE 


\section{WPSReview International on Sustainable Housing and Urban Renewal}

(RI-SHUR)

- La población mundial será mayoritariamente urbana en el año 2050:

En un mundo cada vez más habitado, ${ }^{6}$ la población se concentra cada vez más en zonas urbanas. Según las NN.UU., la población mundial alcanzará los 9.700 millones de habitantes en el año 2050 , y casi el $70 \%$ de ésta vivirá en ciudades. ${ }^{7}$

- Continuidad en el modelo de crecimiento:

En el mundo actual el sistema económico se basa en el crecimiento constante de la producción y el consumo. Este sistema ha traído desarrollo y bienestar a muchas sociedades (principalmente en el mundo occidental). Sin embargo, este modelo resulta insostenible puesto que el ritmo de consumo supera la capacidad de la Tierra. En efecto, se ha estimado que para continuar con el ritmo de crecimiento actual (que, lejos de continuar en realidad está aumentando), el hombre necesitaría 2 planetas para satisfacer sus necesidades en el año $2030 .{ }^{8}$ Además del consumo insostenible de recursos naturales y de energía se añade el aumento de los residuos que producimos, llegando a los 6 millones de toneladas al día en el año 2025 y a los 11 millones de toneladas al día en el $2100 .^{9}$ Todo ello será especialmente grave en las ciudades, donde se concentra la actividad económica del ser humano.

- Cambio climático:

Sin perjuicio de la controversia acerca de sus concretas causas, el cambio climático constituye uno de los problemas centrales del S.XXI y, en este sentido, son numerosos los estudios que, desde diversos campos científicos y con perspectiva medioambiental, acreditan la gravedad de sus efectos, que resultan especialmente intensos en las ciudades. Además, las ciudades son, ellas mismas, factores de aceleración del

\footnotetext{
${ }^{6} \mathrm{http}: / /$ www.un.org/en/development/desa/news/population/2015-report.html.

${ }^{7}$ Globalmente, hay más gente viviendo en zonas urbanas en rurales. En el 2014, el 54\% de la población mundial vivía en ciudades y en 2050 ser prevé que esta cifra alcance el 66\%. https://esa.un.org/unpd/wup/Publications/Files/WUP2014-Highlights.pdf, p. 1, (acceso 20 Noviembre 2016). ${ }^{8}$ http://www.footprintnetwork.org/en/index.php/GFN/page/world footprint/, (acceso 20 Noviembre 2016). 9 https://www.washingtonpost.com/national/health-science/trashing-the-earth-well-soon-make-11-milliontons-of-solid-waste-a-day/2013/11/18/fc243210-4626-11e3-bf0c-cebf37c6f484 story.html, $\quad$ (acceso 20 Noviembre 2016).
}

WPS RI-SHUR, n4, 2016, vol.1, ISSN: 2387-1768

NÚMERO MONOGRÁFICO SOBRE GOBERNANZA URBANA/SPECIAL ISSUE ON GOVERNANCE 


\section{WPSReview International on Sustainable Housing and Urban Renewal}

(RI-SHUR)

calentamiento global de la tierra y de los problemas derivados del mismo que son generados, a su vez, por la emisión de gases de efecto invernadero (GEI).

En este mismo sentido, las ciudades tienen que enfrentarse -muchas lo están haciendo ya- al grave problema de la sobrepoblación que se agravará como consecuencia de los efectos derivados del cambio climático, provocando mayor y más continua inmigración desde las zonas rurales, así como transformaciones radicales del territorio, especialmente en las zonas costeras.

A partir de los datos de Naciones Unidas sobre el crecimiento urbano, es claro que el futuro de cientos de millones de personas se verá afectado por los impactos provocados por la rápida urbanización y agravado por los efectos derivados del cambio climático, por lo que la adaptación es absolutamente necesaria. Esta cuestión es crucial además, porque los efectos del cambio climático ya se están produciendo y porque la mayoría de las ciudades carecen de la capacidad necesaria para responder frente a los mismos de forma eficaz. Particularmente grave es la situación de las ciudades de los países más pobres que son, por ello, también los más vulnerables, por su situación geográfica, por la falta de una planificación adecuada y por la carencia de recursos económicos y políticos para acometer su necesaria transformación. La magnitud de los efectos de las alteraciones de las ciudades dependerá de la forma de asentamientos, las características geográficas y de la naturaleza de la economía local. A pesar de ello, es ya incuestionable que todas las ciudades del mundo serán vulnerables a las inevitables consecuencias del cambio climático. El cambio climático es, por tanto, una realidad, y la gravedad de sus peligrosos efectos, ya inevitables en gran medida, depende de la acción colectiva (en todos los niveles -internacional, regional, nacional, local). Sin embargo, y ahora con mayor claridad, tras los recientes acontecimientos en EE.UU. ${ }^{10}$, se impone el desarrollo de ambiciosas medidas de adaptación frente a los mismos.

- Pérdida del espacio y sentido de lo público

El fenómeno de la globalización ha supuesto una mayor comunicación e interdependencia entre países, generando una dimensión mundial de sus mercados, sociedades y culturas. Sin embargo, a pesar de su globalidad, el mundo actual es cada día más individualista. En efecto, nos encontramos en un momento de la Historia en el que las funciones públicas tradicionales están siendo asumidas por instituciones de

${ }^{10} \mathrm{http}: / /$ internacional.elpais.com/internacional/2016/11/10/actualidad/1478776033 938523.html (acceso 20 Noviembre 2016).

WPS RI-SHUR, nำ, 2016, vol.1, ISSN: 2387-1768

NÚMERO MONOGRÁFICO SOBRE GOBERNANZA URBANA/SPECIAL ISSUE ON GOVERNANCE 
naturaleza bien global, bien privada, provocando así en aquéllas, la pérdida de su rol tradicional y de su sentido de responsabilidad públicos.

De esta forma, parece que la gobernanza actual está integrada por una estructura localglobal (internacional) compleja en la que el neoliberalismo ha favorecido las disparidades sociales y ha transferido el control de la economía desde el sector público al privado, sustituyendo la Ley por el mercado. Esto resulta especialmente relevante en las ciudades, donde se genera la mayoría de la actividad económica.

En necesario, por tanto, rescatar el rol público de la gobernanza urbana para garantizar el desarrollo de políticas públicas dirigidas al fomento de ciudades inclusivas, haciendo de ellas lugares socialmente justos y medioambientalmente sostenibles.

\section{2.- Objetivos de Desarrollo Sostenible}

Los Objetivos de Desarrollo Sostenible (ODS), acordados el 25 de septiembre de $2015^{11}$ señalan 17 metas a alcanzar por todos los países y personas del planeta, y son de amplio alcance, están interrelacionados, se basan en la colaboración y el pragmatismo, y están en conexión con el desarrollo sostenible, incluyendo el crecimiento económico, la inclusión social y la protección del medio ambiente. Entre dichos objetivos está el de "lograr que las ciudades y los asentamientos humanos sean inclusivos, seguros, resilientes y sostenibles" (SDG 11), lo que supone, brevemente: la garantía del acceso a la vivienda y servicios básicos de todas las personas, el acceso a los sistemas de transporte, la protección del patrimonio natural y cultural, la reducción de muertes por desastres naturales, mejorar la calidad de vida de los ciudadanos, y la puesta en marcha de políticas territoriales, de cohesión y financieras, para lograr todo lo anterior. ${ }^{12}$

\section{3.- La Economía Colaborativa como Herramienta de Sostenibilidad/Cohesión}

La economía colaborativa sugiere el uso de la inteligencia del mercado para lograr una sociedad más cooperativa y sostenible. ${ }^{13}$ Resulta común en todos los estudios sobre la economía colaborativa que cuando la información sobre un bien es compartida, el valor

\footnotetext{
${ }^{11} \mathrm{http}: / /$ www.un.org/sustainabledevelopment/ (acceso 20 Noviembre 2016).

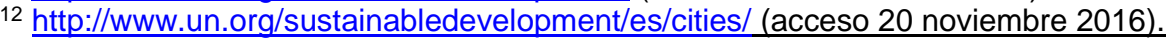

13 Heinrichs, Harald. "Sharing economy: a potential new pathway to sustainability." GAIA-Ecological Perspectives for Science and Society 22, no. 4 (2013): 228-231, p. 229.
} 
de ese bien aumenta para los negocios, para los individuos y para la comunidad. ${ }^{14}$ Pero esta práctica ha supera hoy su significado puramente económico (de mercado) para pasar a informar la actividad de los poderes públicos y, en concreto, tiene el potencial para incorporar nuevas ideas y aproximaciones a la corriente tradicional de pensamiento y formulación de políticas para desarrollar una visión más completa y reforzada del desarrollo sostenible que la utilizada hasta la fecha y facilitar, así, la consecución del SGD número 11 definido por Naciones Unidas.

\section{4.- Propuesta}

Dado el panorama actual y futuro de las ciudades, según se ha descrito brevemente, se impone necesariamente un replanteamiento del sistema, a los efectos de lograr un desarrollo sostenible urbano, en particular, en los países en vías de desarrollo, a partir de la idea de los bienes comunes y de la naturaleza de colaboración que subyace en su naturaleza.

Existen muchas e interesantes propuestas para la definición de las denominadas Smart Cities ${ }^{15}$ pero por los términos en que están definidas, resulta claro que se dirigen a las ciudades del mundo desarrollado.

Ejemplo de ello es el proyecto desarrollado por el Senseable City Lab del MIT, dirigido por Carlo Rotti ${ }^{16}$, y que se basa en 10 ideas fundamentales ${ }^{17}$ :

1) Sobrepoblación, en particular, en las ciudades (utilización de los espacios de forma multifuncional; economía colaborativa);

2) Eficiencia en utilización de recursos (agua, energía, recursos naturales, entre ellos también el espacio -suelo-): eficiencia energética; mejora en la gestión del agua (sistemas de recogida de agua de lluvia); utilización multifuncional de los espacios; gestión eficiente de residuos; información sobre el consumo (behavior change);

\footnotetext{
14 Id.

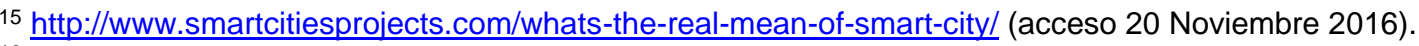

$16 \mathrm{http}: / /$ senseable.mit.edu/ (acceso 20 Noviembre 2016).

17 http://senseable.mit.edu/bitsbricks/ (acceso 20 Noviembre 2016).
}

WPS RI-SHUR, n4, 2016, vol.1, ISSN: 2387-1768

NÚMERO MONOGRÁFICO SOBRE GOBERNANZA URBANA/SPECIAL ISSUE ON GOVERNANCE 
3) Contaminación, heat island effect, impactos cambio climático: aumento de zonas verdes (iniciativa "adopta un árbol"); redes sociales; medidas de mitigación/adaptación al cambio climático;

4) Abuso del coche (transporte privado): planificación de urbes más amables con protagonismo de la bici; bicicletas tecnológicas que dan información sobre su uso; sistemas de transporte colaborativo: coche eléctrico sin conductor; utilización del transporte para conectar zonas aisladas de las ciudades con los centros de actividad (e.g. teleférico Río de Janeiro y Medellín ${ }^{18}$ );

5) Energía: medidas de eficiencia energética; redistribución de energía sobrante; combinación de fuentes de generación de energía: microgrids/paneles en tejados;

6) Consumo lineal de los bienes: reducción del uso de recursos con economía colaborativa: hay muchos elementos/infraestructuras que pueden utilizarse más y mejor si se comparten (e.g. vehículos, edificios);

7) Tráfico urbano: contaminación: movilidad a demanda; combinación transporte público y privado;

8) Conectar la ciudad: ciudades divididas en barrios poco integrados entre sí; creación de nuevas conexiones para evitar la división ricos/pobres; "Modelo de Medellín"19;

9) Uso de electricidad más eficiente y menos contaminante (e.g. semáforos): semáforos e iluminación urbana inteligentes; colocación de sensores urbanos; bombillas LED; $y$

${ }^{18} \mathrm{https}: / / \mathrm{www}$.wired.com/2011/02/st riogondola/ (acceso 20 Noviembre 2016).

${ }_{19}$ https://wearethecityheroes.wordpress.com/2013/01/31/estrategias-urbanas-medellin/ (Acceso 20 Noviembre 2016).

WPS RI-SHUR, no4, 2016, vol.1, ISSN: 2387-1768

NÚMERO MONOGRÁFICO SOBRE GOBERNANZA URBANA/SPECIAL ISSUE ON GOVERNANCE 


\section{WPSReview International on Sustainable Housing and Urban Renewal}

(RI-SHUR)

10) Agricultura urbana: evitar el despilfarro de alimentos; traslado de las explotaciones agrícolas a la ciudad (integración campo/ciudad); huertos urbanos y otras iniciativas: vuelta al origen del ser humano (sentido filosófico. ${ }^{20}$

En efecto, las ideas propuestas se basan en la interconexión de los elementos urbanos a través de la tecnología como fuente de información para lograr un uso más eficiente de los recursos y para lograr un desarrollo más homogéneo e inclusivo de todos los habitantes de las ciudades. Sin embargo, no ha de olvidarse que las ciudades que mayor crecimiento (y transformación) van a experimentar son las de los países en vías de desarrollo, en las que al menos dos de los elementos, tecnología e información, son inaccesibles para la mayoría y de imposible o difícil realización en las (inexistentes, en muchos casos) las políticas públicas del territorio. ${ }^{21}$ Pero además, en los propios países industrializados, existen también muchas poblaciones que, a pesar de estar "conectadas" no podrán acceder al nivel de servicios planteados por las smart cities. Así, las ciudades que quedan al margen de estos avances representan quizá el lugar más adecuado para lograr, a través de los commons, una mayor justicia social.

En este contexto en el que muchas ciudades del mundo quedarán fuera de la dinámica de la modernidad, y en las que las propuestas de acceso a las nuevas tecnologías y a los avances que éstas proponen no son una posibilidad para muchos ciudadanos, los "comunes" pueden ofrecer el marco institucional que sea capaz de poner en cuestión los dominios de la propiedad privada (y del mercado) y del Estado, cambiando la visión dominante del dominio absoluto del sujeto (corporaciones o Estado) sobre el objeto (el territorio y, más general, el medioambiente), para centrarse en la relación equilibrada entre los dos (sujeto-objeto). ${ }^{22}$

\footnotetext{
20 Ejemplos: http://www.swaleny.org/ y http://www.brooklyngrangefarm.com/ (acceso 20 Noviembre 2016).

${ }^{21}$ No ha de olvidarse que el crecimiento de población de las ciudades de países en vías de desarrollo van necesariamente acompañados de problemas de contaminación (del suelo, del agua, del aire), de escasez de suelo para vivienda, de pobreza y segregación, de falta de infraestructuras y de violencia, entre otros, que evidencian la situación de desamparo en la que se encuentran por incapacidad (o falta de interés) del poder público para desarrollar políticas públicas que resuelvan todos estos importantes problemas.

22 Matteri, Ugo. "The State, the market, and Some Preliminary Questions about the Commons (french and English vesrions)." University of California, Hasting College of Law. FRom the SElected Works of Ugo Mattei, March 18, 2011.
}

WPS RI-SHUR, n4, 2016, vol.1, ISSN: 2387-1768

NÚMERO MONOGRÁFICO SOBRE GOBERNANZA URBANA/SPECIAL ISSUE ON GOVERNANCE 
WPSReview International on Sustainable Housing and Urban Renewal

(RI-SHUR)

Para ello, debe responderse a las siguientes preguntas:

1) ¿Cuáles son esos "bienes comunes"? ¿qué incluyen exactamente?

2) ¿Qué papel deben tener los bienes comunes en el desarrollo de las políticas públicas de cohesión territorial y solidaridad social en las ciudades de los países en vías de desarrollo?

3) ¿Qué tipo de políticas colaborativas pueden beneficiar el desarrollo sostenible (en los términos de los SGD) de las ciudades de los países en vías de desarrollo?

4) ¿Cómo se articulan?

WPS RI-SHUR, nำ, 2016, vol.1, ISSN: 2387-1768 